\title{
On differential modular forms and some analytic relations between Eisenstein series $^{1}$
}

\author{
Hossein Movasati \\ Instituto de Matemática Pura e Aplicada, IMPA ${ }^{2}$ \\ Estrada Dona Castorina, 110 \\ 22460-320, Rio de Janeiro, RJ, Brazil \\ E-mail: hossein@impa.br \\ http://www.impa.br/ hossein/
}

\begin{abstract}
In the present article we define the algebra of differential modular forms and we prove that it is generated by Eisenstein series of weight 2,4 and 6. We define Hecke operators on them, find some analytic relations between these Eisenstein series and obtain them in a natural way as coefficients of a family of elliptic curves. The fact that a complex manifold over the moduli of polarized Hodge structures in the case $h^{10}=h^{01}=1$ has an algebraic structure with an action of an algebraic group plays a basic role in all of the proofs.
\end{abstract}

\section{Introduction}

Around 1970 Griffiths introduced the moduli of polarized Hodge structures/the period domain $D$ and described a dream to enlarge $D$ to a moduli space of degenerating polarized Hodge structures. Since in general $D$ is not a Hermitian symmetric domain, he asked for the existence of a certain automorphic cohomology theory for $D$, generalizing the usual notion of automorphic forms on symmetric Hermitian domains. Since then there have been many efforts in the first part of Griffiths's dream (see [7, 12] and the references there) but the second part still lives in darkness.

I was looking for some analytic spaces over $D$ for which one may state Baily-Borel theorem on the unique algebraic structure of quotients of symmetric Hermitian domains by discrete arithmetic groups. I realized that even in the simplest case of Hodge structures, namely $h^{01}=h^{10}=1$, such spaces are not well studied. This led me to the definition of a new class of holomorphic functions on the Poincaré upper half plane which generalize the classical modular forms. Since a differential operator acts on them we call them differential modular forms. These new functions are no longer interpreted as holomorphic sections of a positive line bundle on some compactified moduli curve. Nevertheless, they appear in a natural way as coefficients in families of elliptic curves, analogous to Eisenstein series in the Weierstrass Uniformization Theorem.

Recall the Eisenstein series

$$
g_{k}(z)=a_{k}\left(1+(-1)^{k} \frac{4 k}{B_{k}} \sum_{n \geq 1} \sigma_{2 k-1}(n) e^{2 \pi i z n}\right), \quad k=1,2,3, \quad z \in \mathbb{H},
$$

where $B_{k}$ is the $k$-th Bernoulli number $\left(B_{1}=\frac{1}{6}, B_{2}=\frac{1}{30}, B_{3}=\frac{1}{42}, \ldots\right), \sigma_{i}(n):=\sum_{d \mid n} d^{i}$,

$$
a_{1}=2 \zeta(2) \frac{-1}{2 \pi i}, a_{2}=2 \zeta(4) \frac{60}{(2 \pi i)^{2}}, a_{3}=2 \zeta(6) \frac{-140}{(2 \pi i)^{3}}
$$

\footnotetext{
${ }^{1}$ Keywords: Modular form, Hecke operator, Gauss-Manin connection. Math. classification: 11F11, $14 \mathrm{D} 07$.

${ }^{2}$ Author's address after 1/10/2006.
} 
and $\mathbb{H}:=\{x+i y \in \mathbb{C} \mid y>0\}$ is the Poincaré upper half plane. The most well-known differential modular form, which is not a differential of a modular form, is the Eisenstein series $g_{1}$. The idea of differentiating modular forms and getting new modular forms is old and goes back to Ramanujan. However, the precise definition of differential modular forms has been given recently in [3]. In the present article we give another slightly different definition of differential modular forms (see 2.1 ) over a modular subgroup $\Gamma \subset \operatorname{SL}(2, \mathbb{Z})$. It is based on a canonical behavior of holomorphic functions on the Poincaré upper half plane under the action of $\operatorname{SL}(2, \mathbb{Z})$. This approach has the advantage that it can be generalized to any modular subgroup of $\mathrm{SL}(2, \mathbb{Z})$ but the one in $[3]$ works only in the case of full modular group $\mathrm{SL}(2, \mathbb{Z})$. The set of differential modular forms in the present article is a bigraded $\mathbb{C}$-algebra $M=\sum_{n \in \mathbb{N}_{0}, m \in \mathbb{N}} M_{m}^{n}, M_{m}^{0}$ being the set of classical modular forms of weight $m$, in which the differential operator $\frac{d}{d z}$ maps $M_{m}^{n}$ to $M_{m+2}^{n+1}$. We have $g_{1} \in M_{2}^{1}, g_{2} \in M_{4}^{0}, g_{3} \in M_{6}^{0}$ and we prove:

Theorem 1. The functions $g_{1}, g_{2}, g_{3}$ are algebraically independent and $M$ is freely generated by $g_{1}, g_{2}$ and $g_{3}$ as a $\mathbb{C}$-algebra. For $m \in \mathbb{N}$ and $n \in \mathbb{N}_{0}, M_{m}^{n}$ is the set of homogeneous polynomials of degree $m$ in the graded ring $\mathbb{C}\left[g_{1}, g_{2}, g_{3}\right], \operatorname{deg}\left(g_{i}\right)=2 i, i=1,2,3$ and of degree $2 n$ in $g_{1}$ (note that $\operatorname{deg}\left(g_{1}\right)=2$ ).

The above theorem implies that $M_{m}^{n}=\{0\}$ for $2 n>m$ or $m$ an odd number, and every $f \in M_{m}^{n}$ can be written in a unique way in the form $\sum_{i=0}^{n} f_{i} g_{1}^{i}$, where $f_{i}$ is a modular form of weight $m-2 i$. It generalizes the first theorem in each modular forms book that the algebra of modular forms is freely generated by the Eisenstein series $g_{2}$ and $g_{3}$. Our proof gives us also the Ramanujan relations between the Eisenstein series $g_{i}, i=1,2,3$. We define the action of Hecke operators on $M_{m}^{n}$ and it turns out that this is similar to the case of modular forms:

$$
T_{p} f(z)=p^{m-n-1} \sum_{d \mid p, 0 \leq b \leq d-1} d^{-m} f\left(\frac{p z+b d}{d^{2}}\right), p \in \mathbb{N}, f \in M_{m}^{n} .
$$

Hecke operators of this type appear in particular in the study of the transfer operator from statistical mechanics which plays an important role in the theory of dynamical zeta functions (see [6]). It turns out that the differential operator commutes with Hecke operators (see

$$
g:=\left(g_{1}, g_{2}, g_{3}\right): \mathbb{H} \rightarrow \mathbb{C}^{3}
$$

and

$$
T:=\mathbb{C}^{3} \backslash\left\{\left(t_{1}, t_{2}, t_{3}\right) \in \mathbb{C}^{3} \mid 27 t_{3}^{2}-t_{2}^{3}=0\right\} .
$$

Theorem 2. There are unique analytic functions

$$
B_{1}, B_{2}: T \rightarrow \mathbb{R}, B_{3}: T \rightarrow \mathbb{C}
$$

such that $B_{1}$ does not depend on the variable $t_{1}$ and

$$
B_{1} \circ g(z)=\operatorname{Im}(z), B_{1}\left(t_{1}, t_{2} k^{-4}, t_{3} k^{-6}\right)=B_{1}(t)|k|^{2}
$$

$$
B_{2} \circ g=0, B_{2}\left(t_{1} k^{-2}+k^{\prime} k^{-1}, t_{2} k^{-4}, t_{3} k^{-6}\right)=B_{1}(t)\left|k^{\prime}\right|^{2}+B_{2}(t)\left|k^{-1}\right|^{2}+\operatorname{Im}\left(B_{3}(t) k^{\prime} \overline{k^{-1}}\right)
$$




$$
B_{3} \circ g=1, B_{3}\left(t_{1} k^{-2}+k^{\prime} k^{-1}, t_{2} k^{-4}, t_{3} k^{-6}\right)=B_{3}(t) k \overline{k^{-1}}+2 \sqrt{-1} k \overline{k^{\prime}} B_{1}(t)
$$

for all $k \in \mathbb{C}^{*}$ and $k^{\prime} \in \mathbb{C}$. Moreover, $\left|B_{3}\right|$ restricted to the zero locus of $B_{2}$ is identically one.

Differential modular forms are best viewed as holomorphic functions on the period domain

$$
\mathcal{P}:=\left\{\left(\begin{array}{ll}
x_{1} & x_{2} \\
x_{3} & x_{4}
\end{array}\right) \in \operatorname{GL}(2, \mathbb{C}) \mid \operatorname{Im}\left(x_{1} \overline{x_{3}}\right)>0\right\}
$$

so that they are invariant under the action of $\operatorname{SL}(2, \mathbb{Z})$ from the left on $\mathcal{P}$ and have some compatibility conditions with respect to the action of

$$
G_{0}:=\left\{\left(\begin{array}{cc}
k_{1} & k_{3} \\
0 & k_{2}
\end{array}\right) \mid k_{3} \in \mathbb{C}, k_{1}, k_{2} \in \mathbb{C}^{*}\right\}
$$

from the right on $\mathcal{P}$ (see Proposition [6). In this way Theorem 2 is just the translation of the relations of $g_{i}$ 's with there simple analytic functions on $\mathcal{P}$ (see 2.6) into the coefficient space through the period map (see $\$ 3.2$ ). The action of Hecke operators on differential modular forms is also best viewed in this way. We use a four parameter family of elliptic curves in order to prove our results on differential modular forms and in this way we even obtain a result on the periods of the differential forms of the second type on elliptic curves:

Theorem 3. There is no elliptic curve $E$ and a non-exact differential form of the second type $\omega$ on $E$, both defined over $\overline{\mathbb{Q}}$, such that $\int_{\delta} \omega=0$ for some non-zero topological cycle $\delta \in H_{1}(E, \mathbb{Z})$.

This theorem uses Nesterenko's Theorem (see [13]) on transcendence properties of the values of Eisenstein series. The above theorem for the case in which $\omega$ is of the first kind, is well-known. In this case we can even state it for the field $\mathbb{C}$. However, it is trivially false when $\omega$ is a differential form of the second kind and we allow transcendental coefficients in $\omega$ or the elliptic curve.

The present article stimulates the hope to realize the second part of Griffiths dream with a new formulation. The complex manifold $\mathcal{P}$ can be also introduced over the Griffiths period domain $D$ with an action of an algebraic group $G_{0}$ from the right. Since the differential modular forms on $\mathcal{P}$ are no longer interpreted as sections of positive line bundles over moduli spaces, the question of the existence of a kind of Baily-Borel Theorem for $\mathcal{P}$ arises. In the case of Hodge structures with $h^{01}=h^{10}=1$ we have $D=\mathbb{H}$ and we show that $\mathrm{SL}(2, \mathbb{Z}) \backslash \mathcal{P}$ has a canonical structure of an algebraic quasi-affine variety such that the action of $G_{0}$ from the right is algebraic. More precisely, we prove that $\operatorname{SL}(2, \mathbb{Z}) \backslash \mathcal{P}$ is biholomorphic to $\mathbb{C}^{4} \backslash\left\{t=\left(t_{0}, t_{1}, t_{2}, t_{3}\right) \in \mathbb{C}^{4} \mid t_{0}\left(27 t_{0} t_{3}^{2}-t_{2}^{3}\right)=0\right\}$ and under this biholomorphism the action of $G_{0}$ is given by:

$$
\begin{gathered}
t \bullet g:=\left(t_{0} k_{1}^{-1} k_{2}^{-1}, t_{1} k_{1}^{-1} k_{2}+k_{3} k_{1}^{-1}, t_{2} k_{1}^{-3} k_{2}, t_{3} k_{1}^{-4} k_{2}^{2}\right), \\
t=\left(t_{0}, t_{1}, t_{2}, t_{3}\right) \in \mathbb{C}^{4}, g=\left(\begin{array}{cc}
k_{1} & k_{3} \\
0 & k_{2}
\end{array}\right) \in G_{0} .
\end{gathered}
$$


The mentioned biholomorphism is given by the period map (see 3.2). Using the methods of present article, one can describe the dynamics of the holomorphic foliation induced by the Ramanujan's relations. This will be discussed in another paper.

Let us now explain the structure of this article. \$2 is devoted to the definition of differential modular forms and the action of Hecke operators on them. 3 is devoted to the calculation of the Gauss-Manin connection of a family of elliptic curves. In this section we prove that the period map is a biholomorphism and then we take its inverse and obtain the Ramanujan relations. Finally, 4 is devoted to the proof of theorems announced in the Introduction.

Acknowledgment: The main ideas of this paper took place in my mind when I was visiting Prof. Sampei Usui at Osaka University. Here I would like to thank him for encouraging me to study Hodge theory and for his help to understand it. I would like to thank Prof. Karl-Hermann Neeb for his interest and careful reading of the present article. I would like to thank the referee of the present article who made useful comments on the first draft of this text and introduced me with the reference [8] in which the notion of a differential modular form with the name quasi modular form is introduced and Theorem 1 is proved by means of algebraic methods. The alternative proof presented here by means of the period map might be useful for further analyzing the differential modular forms and their relations with elliptic curves.

\section{$2 \quad M_{m}^{n}$-functions}

In this section we use the notations $A=\left(\begin{array}{ll}a_{A} & b_{A} \\ c_{A} & d_{A}\end{array}\right) \in \mathrm{SL}(2, \mathbb{R})$ and

$$
\begin{gathered}
I=\left(\begin{array}{ll}
1 & 0 \\
0 & 1
\end{array}\right), T=\left(\begin{array}{ll}
1 & 1 \\
0 & 1
\end{array}\right), Q=\left(\begin{array}{cc}
0 & -1 \\
1 & 0
\end{array}\right), \\
x=\left(\begin{array}{ll}
x_{1} & x_{2} \\
x_{3} & x_{4}
\end{array}\right), g=\left(\begin{array}{cc}
k_{1} & k_{2} \\
0 & k_{3}
\end{array}\right), x, g \in \mathrm{GL}(2, \mathbb{C}) .
\end{gathered}
$$

When there is no confusion we will simply write $A=\left(\begin{array}{ll}a & b \\ c & d\end{array}\right)$. We denote by $\mathbb{H}$ the Poincaré upper half plane and

$$
\mathrm{j}(A, z):=c_{A} z+d_{A}
$$

For $A \in \mathrm{SL}(2, \mathbb{R})$ and $m \in \mathbb{Z}$ we use the slash operator

$$
\left.f\right|_{m} A=(\operatorname{det} A)^{m-1} \mathrm{j}(A, z)^{-m} f(A z) .
$$

For a ring $R$ we denote by $\operatorname{Mat}_{p}(2, R)$ the set of $2 \times 2$-matrices in $R$ with the determinant $p$.

\subsection{Definitions}

In this section we define the notion of an $M_{m}^{n}$-function. For $n=0$ an $M_{m}^{0}$-function is a classical modular form of weight $m$ on $\mathbb{H}$ (see bellow). A holomorphic function $f$ on $\mathbb{H}$ is called $M_{m}^{n}$ if the following two conditions are satisfied: 
1. There are holomorphic functions $f_{i}, i=0,1, \ldots, n$ on $\mathbb{H}$ such that

$$
\left.f\right|_{m} A=\sum_{i=0}^{n}\left(\begin{array}{l}
n \\
i
\end{array}\right) c_{A}^{i} \mathrm{j}(A, z)^{-i} f_{i}, \forall A \in \mathrm{SL}(2, \mathbb{Z}) .
$$

2. $f_{i}, i=0,1,2, \ldots, n$ have finite growths when $\operatorname{Im}(z)$ tends to $+\infty$, i.e.

$$
\lim _{\operatorname{Im}(z) \rightarrow+\infty} f_{i}(z)=a_{i, \infty}<\infty, a_{i, \infty} \in \mathbb{C} .
$$

The above definition can be made using a subgroup $\Gamma \subset \mathrm{SL}(2, \mathbb{Z})$. In this article we mainly deal with full differential modular forms, i.e. the case $\Gamma=\mathrm{SL}(2, \mathbb{Z})$. We will also denote by $M_{m}^{n}$ the set of $M_{m}^{n}$-functions and we set

$$
M:=\sum_{m \in \mathbb{Z}, n \in \mathbb{N}_{0}} M_{m}^{n}
$$

For an $f \in M_{m}^{n}$ we have $\left.f\right|_{m} I=f_{0}$ and so $f_{0}=f$. We have also $\left.f\right|_{m} T=f$ and so we can write the Fourier expansion of $f$ at infinity

$$
f=\sum_{n=-N}^{+\infty} a_{n} q^{n}, a_{n} \in \mathbb{C}, N=0,1,2, \ldots, \infty, q=e^{2 \pi i z} .
$$

The growth condition on $f$ implies that $N=0$. Note that for an $M_{m}^{n}$-function $f$ the associated functions $f_{i}$ are unique. To see this fix $z$ and consider the right hand side of (10) as a polynomial in $c_{A} \mathrm{j}(A, z)^{-1}$ with coefficients $\left(\begin{array}{c}n \\ i\end{array}\right) f_{i}$. Since $A$ is an arbitrary element of $\operatorname{SL}(2, \mathbb{Z})$ and a one variable polynomial has a finite number of roots, we conclude that $f_{i}$ 's are unique.

Proposition 1. If $f$ is $M_{m}^{n}$-function with the associated functions $f_{i}$ then $f_{i}$ is an $M_{m-2 i}^{n-i}$ function with the associated functions $f_{i j}:=f_{i+j}, j=0,1, \ldots, n-i$, i.e.

$$
\left.f_{i}\right|_{m-2 i} A=\sum_{j=0}^{n-i}\left(\begin{array}{c}
n-i \\
j
\end{array}\right) c_{A}^{j} \mathrm{j}(A, z)^{-j} f_{i j}, \forall A \in \mathrm{SL}(2, \mathbb{Z}), f_{i j}=f_{i+j} .
$$

Proof. For $A, B \in \mathrm{SL}(2, \mathbb{Z})$ we have

$$
\begin{aligned}
f(A B z) & =\mathrm{j}(A B, z)^{m} \sum_{i=0}^{n}\left(\begin{array}{c}
n \\
i
\end{array}\right) c_{A B}^{i} \mathrm{j}(A B, z)^{-i} f_{i}(z) \\
& =\mathrm{j}(A B, z)^{m} \sum_{i=0}^{n}\left(\begin{array}{c}
n \\
i
\end{array}\right)\left(c_{A B} \mathrm{j}(B, z)\right)^{i} \mathrm{j}(B, z)^{-i} \mathrm{j}(A B, z)^{-i} f_{i}(z) \\
& =\mathrm{j}(A B, z)^{m} \sum_{i=0}^{n} \sum_{j=0}^{i}\left(\begin{array}{c}
n \\
i
\end{array}\right)\left(\begin{array}{c}
i \\
j
\end{array}\right) \mathrm{j}(A B, z)^{j} c_{B}^{j} c_{A}^{i-j} \mathrm{j}(B, z)^{-i} \mathrm{j}(A B, z)^{-i} f_{i}(z) \\
& =\mathrm{j}(A B, z)^{m} \sum_{r=0}^{n} \sum_{s=0}^{n-r}\left(\begin{array}{c}
n \\
r+s
\end{array}\right)\left(\begin{array}{c}
r+s \\
s
\end{array}\right) c_{B}^{s} c_{A}^{r} \mathrm{j}(B, z)^{-r-s} \mathrm{j}(A B, z)^{-r} f_{r+s}(z) \\
& =\mathrm{j}(A, B z)^{m} \sum_{r=0}^{n}\left(\begin{array}{c}
n \\
r
\end{array}\right) c_{A}^{r} \mathrm{j}(A, B z)^{-r}\left(\mathrm{j}(B, z)^{m-2 r} \sum_{s=0}^{n-r}\left(\begin{array}{c}
n-r \\
s
\end{array}\right) c_{B}^{s} \mathrm{j}(B, z)^{-s} f_{r+s}(z)\right)
\end{aligned}
$$


In the first equality we have used (10). In the third equality we have used

$$
\mathrm{j}(A B, z) c_{B}+\operatorname{det}(B) c_{A}=c_{A B \mathrm{j}}(B, z), \forall A, B \in \mathrm{GL}(2, \mathbb{R}) .
$$

In the fourth equality we have changed the counting parameters: $r=i-j, s=j, 0 \leq$ $r+s \leq n$. In the fifth equality we have used

$$
\mathrm{j}(A B, z)=\mathrm{j}(A, B z) \mathrm{j}(B, z) .
$$

From another side

$$
\begin{aligned}
f(A B z) & =f(A(B z)) \\
& =\mathrm{j}(A, B z)^{m} \sum_{r=0}^{n}\left(\begin{array}{l}
n \\
r
\end{array}\right) c_{A}^{r} \mathrm{j}(A, B z)^{-r} f_{r}(B z) .
\end{aligned}
$$

Since the holomorphic functions associated to $f$ are unique, we conclude that

$$
f_{r}(B z)=\mathrm{j}(B, z)^{m-2 r} \sum_{s=0}^{n-r}\left(\begin{array}{c}
n-r \\
s
\end{array}\right) c_{B}^{s} \mathrm{j}(B, z)^{-s} f_{r+s}(z), \forall B \in \mathrm{SL}(2, \mathbb{Z}), r=0,1, \ldots, n .
$$

It is useful to define

$$
f \|_{m} A:=(\operatorname{det} A)^{m-n-1} \sum_{i=0}^{n}\left(\begin{array}{c}
n \\
i
\end{array}\right) c_{A^{-1} \mathrm{j}}(A, z)^{i-m} f_{i}(A z), A \in \mathrm{GL}(2, \mathbb{R}), f \in M_{m}^{n} .
$$

The factor $\operatorname{det} A$ is introduced because of Hecke operators (see $\$ 2.3$ ). The equalities (10) is written in the form

$$
f=f \|_{m} A, \forall A \in \mathrm{SL}(2, \mathbb{Z})
$$

(we have substituted $A^{-1} z$ for $z$ and then $A^{-1}$ for $A$ ). Since $f \|_{m} A, A \in \mathrm{GL}(2, \mathbb{Z}), f \in M_{m}^{n}$ may not be in $M$ and it is defined using the associated functions of $f$, it does not make sense to say that $\|_{m}$ is an action of $\mathrm{GL}(2, \mathbb{R})$ on $M_{m}^{n}$ from the right. However, we have the following proposition:

Proposition 2. We have

$$
f\left\|_{m} A=f\right\|_{m}(B A), \forall A \in \mathrm{GL}(2, \mathbb{R}), B \in \mathrm{SL}(2, \mathbb{Z}), \quad f \in M_{m}^{n}
$$

Proof. The proof is similar to the the proof of Proposition [1. The term $(\operatorname{det} A)^{n-m+1} f \|_{m} A(z)$ 
is equal to:

$$
\begin{aligned}
& =\sum_{i=0}^{n}\left(\begin{array}{c}
n \\
i
\end{array}\right) c_{A^{-1}}^{i} \mathrm{j}(A, z)^{i-m} f_{i}\left(B^{-1} B A z\right) \\
& =\sum_{i=0}^{n} \sum_{j=0}^{n-i}\left(\begin{array}{c}
n \\
i
\end{array}\right)\left(\begin{array}{c}
n-i \\
j
\end{array}\right) c_{A^{-1}}^{i} c_{B^{-1}}^{j} \mathrm{j}(A, z)^{i-m} \mathrm{j}\left(B^{-1}, B A z\right)^{m-2 i-j} f_{i+j}(B A z) \\
& =\sum_{r=0}^{n} \sum_{s=0}^{r}\left(\begin{array}{c}
n \\
s
\end{array}\right)\left(\begin{array}{c}
n-s \\
r-s
\end{array}\right) c_{A^{-1}}^{s} c_{B^{-1}}^{r-s} \mathrm{j}(A, z)^{s-m} \mathrm{j}\left(B^{-1}, B A z\right)^{m-r-s} f_{r}(B A z) \\
& =\sum_{r=0}^{n}\left(\begin{array}{c}
n \\
r
\end{array}\right) \mathrm{j}(B A z, z)^{r-m} f_{r}(B A z) \mathrm{j}(A, z)^{-r}\left(\sum_{s=0}^{r}\left(\begin{array}{l}
r \\
s
\end{array}\right) \mathrm{j}(B A, z)^{s} c_{A^{-1}}^{s} c_{B^{-1}}^{r-s}\right) \\
& =\sum_{r=0}^{n}\left(\begin{array}{c}
n \\
r
\end{array}\right) \mathrm{j}(B A, z)^{r-m} f_{r}(B A z) \mathrm{j}(A, z)^{-r}\left(\mathrm{j}(B A, z) c_{A^{-1}}+c_{B^{-1}}\right)^{r} \\
& =\sum_{r=0}^{n}\left(\begin{array}{c}
n \\
r
\end{array}\right) \mathrm{j}(B A, z)^{r-m} c_{(B A)^{-1}}^{r} f_{r}(B A z)=(\operatorname{det} A)^{n-m+1}\left(f \|_{m} B A\right)(z)
\end{aligned}
$$

\subsection{Algebra of $M_{m}^{n}$-functions}

Recall the Eisenstein series (11) and

$$
\begin{gathered}
\Delta(z):=\left(27 g_{3}^{2}(z)-g_{2}^{3}(z)\right)=-\left(\frac{2 \pi i}{12}\right)^{6} q \prod_{n=1}^{\infty}\left(1-q^{n}\right)^{24}=q-24 q^{2}+252 q^{3}+\cdots, \\
j(z):=\frac{g_{2}^{3}(z)}{-\Delta(z)}=q^{-1}+744+196884 q+\cdots
\end{gathered}
$$

Note that $\zeta(2)=\frac{\pi^{2}}{6}, \zeta(4)=\frac{\pi^{4}}{90}, \zeta(6)=\frac{\pi^{6}}{945}$ and so

$$
p_{\infty}:=\left(a_{1}, a_{2}, a_{3}\right)=\left(\frac{2 \pi i}{12}, 12\left(\frac{2 \pi i}{12}\right)^{2}, 8\left(\frac{2 \pi i}{12}\right)^{3}\right),
$$

where $a_{i}$ 's are defined in (2). For $k \geq 2$ one can write

$$
g_{k}(z)=s_{k} \sum_{0 \neq(m, n) \in \mathbb{Z}^{2}} \frac{1}{(n+m z)^{2 k}} \in M_{2 k}^{0},
$$

where $s_{2}=\frac{60}{(2 \pi i)^{2}}$ and $s_{3}=\frac{-140}{(2 \pi i)^{3}}$. The Eisenstein series $g_{1}$ satisfies

$$
\left.g_{1}\right|_{2} A-g_{1}=c \mathrm{j}(A, z)^{-1}, A \in \mathrm{SL}(2, \mathbb{Z})
$$

and so $g_{1} \in M_{2}^{1}$ (see for instance [1] p. 69). The following proposition describes the algebraic structure of $M_{m}^{n}$ :

Proposition 3. The followings are true:

1. For an $f \in M_{m}^{1}$ the function $z\left(z^{-m} f\left(\frac{-1}{z}\right)-f(z)\right)$ is in $M_{m-2}^{0}$, i.e. it is a modular form of weight $m-2$. 
2. $M_{2}^{1}$ is a one dimensional $\mathbb{C}$-vector space generated by $g_{1}$.

3. If $n \leq n^{\prime}$ then $M_{m}^{n} \subset M_{m}^{n^{\prime}}$ and

$$
M_{m}^{n} M_{m^{\prime}}^{n^{\prime}} \subset M_{m+m^{\prime}}^{n+n^{\prime}}, M_{m}^{n}+M_{m}^{n^{\prime}}=M_{m}^{n^{\prime}}
$$

4. For a modular form $f$ of weight $m$ we have $f\left(g_{1}\right)^{n} \in M_{2 n+m}^{n}$.

Proof. The first item is a direct consequence of Proposition 1 and the definition of a $M_{2}^{1}$-function applied to $A=Q$ :

$$
z^{-m} f\left(\frac{-1}{z}\right)=\left.f\right|_{m} Q=f+z^{-1} f_{1}(z), f_{1} \in M_{m-2}^{0} .
$$

Since the modular forms of weight 0 are constant functions, every $f \in M_{2}^{1}$ satisfies: $\left.f\right|_{2} A=f+\operatorname{rcj}(A, z)^{-1}, \forall A \in \mathrm{SL}(2, \mathbb{Z})$ for some constant $r \in \mathbb{C}$. This and (16) implies that $f-r g_{2}$ is a modular form of weight 2 . Since there is no non-zero modular form of weight 2 we conclude that $M_{2}^{1}$ is generated by $g_{1}$.

If $f \in M_{m}^{n}$ with the associated functions $f_{i}, i=0,1, \ldots, n$ then $f \in M_{m}^{n^{\prime}}$ with the associated functions $f_{i}, i=0,1, \ldots, n, f_{i}=0, i=n+1, \ldots, n^{\prime}$. If $f \in M_{m}^{n}$ and $g \in M_{m^{\prime}}^{n^{\prime}}$ with the associated functions $f_{i}, i=0,1, \ldots, n$ (resp. $\left.g_{i}, i=0,1, \ldots, n^{\prime}\right)$ then for $A \in \mathrm{SL}(2, \mathbb{Z})$

$$
\begin{aligned}
\left.f g\right|_{m+m^{\prime}} A & =\left.\left.f\right|_{m} A \cdot g\right|_{m^{\prime}} A \\
& =\left(\sum_{i=0}^{n}\left(\begin{array}{c}
n \\
i
\end{array}\right) c_{A}^{i} \mathrm{j}(A, z)^{-i} f_{i}\right)\left(\sum_{j=0}^{n^{\prime}}\left(\begin{array}{c}
n^{\prime} \\
j
\end{array}\right) c_{A}^{j} \mathrm{j}(A, z)^{-j} g_{j}\right) \\
& =\sum_{r=0}^{n+n^{\prime}}\left(\begin{array}{c}
n+n^{\prime} \\
r
\end{array}\right) c_{A}^{r} \mathrm{j}(A, z)^{-r}\left(\sum_{s=0}^{r} \frac{\left(\begin{array}{c}
n \\
s
\end{array}\right)\left(\begin{array}{c}
n^{\prime} \\
r-s
\end{array}\right)}{\left(\begin{array}{c}
n+n^{\prime} \\
r
\end{array}\right)} f_{s} g_{r-s}\right)
\end{aligned}
$$

which implies that $f g \in M_{m+m^{\prime}}^{n+n^{\prime}}$. Now if $m=m^{\prime}$ then by the discussion at the beginning of this paragraph we can assume that $n=n^{\prime}$. Now, $f+g \in M_{m}^{n^{\prime}}$ with the associated functions $f_{i}+g_{i}, i=0,1, \ldots, n^{\prime}$.

The fourth item is a consequence of item 3 . It was the main idea behind the definition of $M_{m}^{n}$.

The following proposition shows that $M$ is in fact a differential algebra.

Proposition 4. For $f \in M_{m}^{n}$ we have $\frac{d f}{d z} \in M_{m+2}^{n+1}$ and

$$
\frac{d\left(f \|_{m} A\right)}{d z}=\frac{d f}{d z} \|_{m+2} A, \forall A \in \mathrm{GL}(2, \mathbb{R})
$$


Proof. For $A \in \mathrm{GL}(2, \mathbb{Z})$ with $\operatorname{det}(A)=p$ the term $\frac{d\left(f \|_{m} A\right)}{d z}$ is equal to:

$$
\begin{aligned}
& =p^{m-n}\left(\sum_{i=0}^{n}\left(\begin{array}{c}
n \\
i
\end{array}\right) c_{A^{-1}}^{i}\left((m-i) c_{A^{-1}} \mathrm{j}(A, z)^{i-1-m} f_{i}(A z)+\mathrm{j}(A, z)^{i-m-2} \frac{d f_{i}}{d z}(A z)\right)\right) \\
& =p^{m-n}\left(\sum_{i=1}^{n+1}\left(\begin{array}{c}
n \\
i-1
\end{array}\right) c_{A^{-1} \mathrm{j}}^{i}(A, z)^{i-2-m}(m-i+1) f_{i-1}(A z)\right. \\
& \left.+\sum_{i=0}^{n}\left(\begin{array}{c}
n \\
i
\end{array}\right) c_{A^{-1} \mathrm{j}}(A, z)^{i-2-m} \frac{d f_{i}}{d z}(A z)\right) \\
& =p^{m-n}\left(\sum_{i=0}^{n+1}\left(\begin{array}{c}
n+1 \\
i
\end{array}\right) c_{A^{-1}}^{i}(A, z)^{i-2-m} \tilde{f}_{i}(A z)\right) \text {, }
\end{aligned}
$$

where

$$
\tilde{f}_{i}=\frac{i(m-i+1)}{n+1} f_{i-1}+\frac{n+1-i}{n+1} \frac{d f_{i}}{d z}, i=0,1, \ldots, n+1, f_{-1}=f_{n+1}:=0 .
$$

For $A \in \mathrm{SL}(2, \mathbb{Z})$ we have $f=f \|_{m} A$ and so $\frac{d f}{d z}=\frac{d\left(f \|_{m} A\right)}{d z}$. This and the above equalities imply that $\frac{d f}{d z}$ is an $M_{m+2}^{n+1}$-function with the associated $M_{m-2 i}^{n-i}$-functions $\tilde{f}_{i}, i=$ $0,1,2, \ldots, n+1$. The growth condition on $\tilde{f}_{i}$ 's follows from

$$
\frac{d f}{d z}=2 \pi i q \frac{d f}{d q}
$$

For an arbitrary $A \in \mathrm{GL}(2, \mathbb{R})$, (17) follows from the equalities at the beginning of the proof.

The relations between the $g_{i}, i=1,2,3$ and their derivatives are given by the Ramanujan's equalities:

$$
\frac{d g_{1}}{d z}=g_{1}^{2}-\frac{1}{12} g_{2}, \frac{d g_{2}}{d z}=4 g_{1} g_{2}-6 g_{3}, \frac{d g_{3}}{d z}=6 g_{1} g_{3}-\frac{1}{3} g_{2}^{2}
$$

(see for instance [10, 13]). The proof of Theorem 1 will contain a new geometric proof of these equalities.

\section{$2.3 \quad$ Hecke operators}

For $p \in \mathbb{N}$ let $\operatorname{SL}(2, \mathbb{Z}) \backslash \operatorname{Mat}_{p}(2, \mathbb{Z})=\left\{\left[A_{1}\right],\left[A_{2}\right], \ldots,\left[A_{s}\right]\right\}$. We define the $p$-th Hecke operator in the following way

$$
T_{p} f:=\sum_{k=1}^{s} f \|_{m} A_{k}, f \in M_{m}^{n} .
$$

Proposition 2 implies that the above definition does not depend on the choice of $A_{k}$ in the class $\left[A_{k}\right]$. Form Proposition 4 one can deduce that the differential operator $\frac{d}{d z}$ commutes with the Hecke operator $T_{p}$.

Proposition 5. $T_{p}$ defines a map from $M_{m}^{n}$ to itself. 
This will be proved in $\$ 2.5$ One can take

$$
\tilde{T}_{p}:=\sum_{d \mid p, 0 \leq b \leq d-1}\left(\begin{array}{ll}
\frac{p}{d} & b \\
0 & d
\end{array}\right) \in \mathbb{Z}\left[\operatorname{Mat}_{p}(2, \mathbb{Z})\right]
$$

and since for matrices $\left(\begin{array}{ll}a & b \\ 0 & d\end{array}\right)$ the slash operator $\left.\right|_{m}$ is $p^{n}$ times $\|_{m}$ we have $T_{p} f=$ $\left.p^{-n} f\right|_{m} \tilde{T}_{p}$ and we get the expression (3) in the Introduction. Similar to the case of modular forms (see [1] §6) one can check that

$$
T_{p} \circ T_{q}=\sum_{d \mid(p, q)} d^{m-n-1} T \frac{p q}{d^{2}} .
$$

\subsection{The period domain}

The group $\operatorname{SL}(2, \mathbb{Z})$ acts from the left on the period domain $\mathcal{P}$ defined in (7) and $G_{0}$ in (8) acts from the right. We consider a holomorphic function on

$$
\mathcal{L}:=\operatorname{SL}(2, \mathbb{Z}) \backslash \mathcal{P}
$$

as a holomorphic function

$$
f: \mathcal{P} \rightarrow \mathbb{C}, \text { holomorphic satisfying }, f(A z)=f(z), \forall A \in \mathrm{SL}(2, \mathbb{Z}), z \in \mathcal{P} .
$$

The determinant function is such a function. The Poincaré upper half plane $\mathbb{H}$ is embedded in $\mathcal{P}$ in the following way:

$$
z \rightarrow \tilde{z}=\left(\begin{array}{cc}
z & -1 \\
1 & 0
\end{array}\right)
$$

We denote by $\tilde{\mathbb{H}}$ the image of $\mathbb{H}$ under this map. For a function $f$ on $\mathbb{H}$ we denote by $\tilde{f}$ the corresponding function on $\tilde{\mathbb{H}}$.

Proposition 6. There is a unique map

$$
\phi: M \rightarrow \mathcal{O}(\mathcal{P}), f \mapsto \phi(f)=F
$$

of the algebra of $M$-functions into the algebra of holomorphic functions on $\mathcal{P}$ such that

1. For all $f \in M$ the restriction of $F$ to $\tilde{\mathbb{H}}$ is equal to $\tilde{f}$.

2. For all $f \in M$ the holomorphic function $F$ is $\mathrm{SL}(2, \mathbb{Z})$ invariant.

3. We have

$$
F(x \cdot g)=k_{2}^{n} k_{1}^{n-m} \sum_{i=0}^{n}\left(\begin{array}{l}
n \\
i
\end{array}\right) k_{3}^{i} k_{2}^{-i} F_{i}(x), \forall x \in \mathcal{P}, g \in G_{0}
$$

where $F_{i}=\phi\left(f_{i}\right)$.

Conversely, every holomorphic function $F$ on $\mathcal{P}$ which is left $\mathrm{SL}(2, \mathbb{Z})$-invariant and satisfies (19) for some holomorphic functions $F_{i}$ on $\mathcal{P}$ such that the restriction of $F_{i}$ 's to $\tilde{\mathbb{H}}$ have finite growths at infinity is of the form $F=\phi(f)$ for some $f \in M_{m}^{n}$. 
Proof. We have

$$
\left(\begin{array}{ll}
x_{1} & x_{2} \\
x_{3} & x_{4}
\end{array}\right)=\left(\begin{array}{cc}
\frac{x_{1}}{x_{3}} & -1 \\
1 & 0
\end{array}\right)\left(\begin{array}{cc}
x_{3} & x_{4} \\
0 & \frac{\operatorname{det}(x)}{x_{3}}
\end{array}\right) .
$$

Therefore, we expect $F$ to be defined by

$$
F(x)=F\left(\left(\begin{array}{cc}
\frac{x_{1}}{x_{3}} & -1 \\
1 & 0
\end{array}\right)\left(\begin{array}{cc}
x_{3} & x_{4} \\
0 & \frac{\operatorname{det}(x)}{x_{3}}
\end{array}\right)\right):=x_{3}^{-m} \operatorname{det}(x)^{n} \sum_{i=0}^{n}\left(\begin{array}{c}
n \\
i
\end{array}\right) x_{4}^{i} x_{3}^{i} \operatorname{det}(x)^{-i} f_{i}\left(\frac{x_{1}}{x_{3}}\right) .
$$

Let us prove that the function $f \mapsto F=\phi(f)$ satisfies the items 1,2 and 3. For $x=\tilde{z}, z \in \mathbb{H}$ we have $x_{4}=0$ and so $F(x)=f_{0}(z)=f(z)$. This proves the first item.

By the definition of $F$ one can rewrite (10) in the form

$$
f\left(A \frac{x_{1}}{x_{3}}\right)=\left(c x_{1}+d x_{3}\right)^{m-n} F\left(\begin{array}{cc}
x_{1} & -d \\
x_{3} & c
\end{array}\right)
$$

where $A=\left(\begin{array}{ll}a & b \\ c & d\end{array}\right) \in \mathrm{SL}(2, \mathbb{Z})$.

Now, we prove item 3 . Let

$$
g^{\prime}=\left(\begin{array}{cc}
k_{1}^{\prime} & k_{3}^{\prime} \\
0 & k_{2}^{\prime}
\end{array}\right):=\left(\begin{array}{cc}
x_{3} & x_{4} \\
0 & \frac{\operatorname{det}(x)}{x_{3}}
\end{array}\right) .
$$

$$
\begin{aligned}
\text { RHS of (19) } & =k_{2}^{n} k_{1}^{n-m} \sum_{i=0}^{n}\left(\begin{array}{c}
n \\
i
\end{array}\right) k_{3}^{i} k_{2}^{-i} F_{i}(x) \\
& =\left(k_{2} k_{2}^{\prime}\right)^{n}\left(k_{1} k_{1}^{\prime}\right)^{n-m} \sum_{i=0}^{n} \sum_{j=0}^{n-i}\left(\begin{array}{c}
n \\
i
\end{array}\right)\left(\begin{array}{c}
n-i \\
j
\end{array}\right) k_{3}^{i} k_{2}^{-i} k_{2}^{\prime-i} k_{1}^{\prime i} k_{3}^{\prime j} k_{2}^{\prime-j} f_{i+j}\left(\frac{x_{1}}{x_{3}}\right) \\
& =\left(k_{2} k_{2}^{\prime}\right)^{n}\left(k_{1} k_{1}^{\prime}\right)^{n-m} \sum_{r=0}^{n} \sum_{s=0}^{r}\left(\begin{array}{c}
n \\
s
\end{array}\right)\left(\begin{array}{c}
n-s \\
r-s
\end{array}\right) k_{3}^{s} k_{2}^{-s} k_{2}^{\prime-s} k_{1}^{\prime s} k_{3}^{\prime r-s} k_{2}^{\prime-r+s} f_{r}\left(\frac{x_{1}}{x_{3}}\right) \\
& =\left(k_{2} k_{2}^{\prime}\right)^{n}\left(k_{1} k_{1}^{\prime}\right)^{n-m} \sum_{r=0}^{n}\left(\begin{array}{c}
n \\
r
\end{array}\right)\left(\sum_{s=0}^{r}\left(\begin{array}{c}
r \\
s
\end{array}\right)\left(k_{2} k_{3}^{\prime}\right)^{r-s}\left(k_{3} k_{1}^{\prime}\right)^{s}\right)\left(k_{2} k_{2}^{\prime}\right)^{-r} f_{r}\left(\frac{x_{1}}{x_{3}}\right) \\
& =\left(k_{2} k_{2}^{\prime}\right)^{n}\left(k_{1} k_{1}^{\prime}\right)^{n-m} \sum_{r=0}^{n}\left(\begin{array}{c}
n \\
r
\end{array}\right)\left(k_{2} k_{3}^{\prime}+k_{3} k_{1}^{\prime}\right)^{r}\left(k_{2} k_{2}^{\prime}\right)^{-r} f_{r}\left(\frac{x_{1}}{x_{3}}\right) \\
& =F\left(\left(\begin{array}{cc}
z & -1 \\
1 & 0
\end{array}\right) g^{\prime} g\right)=F(x g)
\end{aligned}
$$

In the second equality we have used the definition of $F_{i}=\phi\left(f_{i}\right)$ as in (21) and the fact that the associated functions of $f_{i}$ are $f_{i+j}, j=0,1, \ldots, n-i$, where $f_{i}$ 's are associated functions of $f$ (see Proposition 11). In the third equality we have changed the counting parameters: $s=i, r=i+j$. The fifth equality is just the expansion of $\left(k_{2} k_{3}^{\prime}+k_{3} k_{1}^{\prime}\right)^{r}$. The sixth equality is by the definition of $F=\phi(f)$. 
Let us now prove the second item. We have to prove that $F(A x)=F(x), \forall A \in$ $\mathrm{SL}(2, \mathbb{Z})$ : The term $F(A x)$ is equal to

$$
\begin{aligned}
= & \left(c x_{1}+d x_{3}\right)^{-m} \operatorname{det}(x)^{n} \sum_{i=0}^{n}\left(\begin{array}{c}
n \\
i
\end{array}\right)\left(c x_{2}+d x_{4}\right)^{i}\left(c x_{1}+d x_{3}\right)^{i} \operatorname{det}(x)^{-i} f_{i}\left(A \frac{x_{1}}{x_{3}}\right) \\
= & \left(c x_{1}+d x_{3}\right)^{-m} \operatorname{det}(x)^{n} \\
& \sum_{i=0}^{n}\left(\begin{array}{c}
n \\
i
\end{array}\right)\left(c x_{2}+d x_{4}\right)^{i}\left(c x_{1}+d x_{3}\right)^{i} \operatorname{det}(x)^{-i}\left(c x_{1}+d x_{3}\right)^{m-2 i-(n-i)} F_{i}\left(\begin{array}{cc}
x_{1} & -d \\
x_{3} & c
\end{array}\right) \\
= & F\left(\left(\begin{array}{cc}
x_{1} & -d \\
x_{3} & c
\end{array}\right)\left(\begin{array}{cc}
1 & \frac{c x_{2}+d x_{4}}{c x_{1}+d x_{3}} \\
0 & \frac{\operatorname{det}(x)}{c x_{1}+d x_{3}}
\end{array}\right)\right)=F(x) .
\end{aligned}
$$

In the second equality we have used the fact that $f_{i} \in M_{m-2 i}^{n-i}$ and the corresponding equality (22). In the third equality we have used the third item of Proposition 6 ,

We have finished the proof of the fact that $F=\phi(f)$ has the desired properties. Now, let $F$ satisfy 2,3 and its restriction to $\tilde{\mathbb{H}}$ has a finite growth at infinity. Put $f=\left.F\right|_{\tilde{\mathbb{H}}}$ and $f_{i}:=\left.F_{i}\right|_{\tilde{H}}$. We are going to prove that $f$ satisfies (10) with the associated functions $f_{i}$ 's and so $f \in M_{m}^{n}$. First, we note that

$$
\left(\begin{array}{ll}
a & b \\
c & d
\end{array}\right)\left(\begin{array}{cc}
z & -1 \\
1 & 0
\end{array}\right)=\left(\begin{array}{cc}
A z & -1 \\
1 & 0
\end{array}\right)\left(\begin{array}{cc}
\mathrm{j}(A, z) & -c \\
0 & \mathrm{j}(A, z)^{-1} \operatorname{det}(A)
\end{array}\right), A \in \mathrm{GL}(2, \mathbb{R}) .
$$

Now

$$
\begin{aligned}
f(A z) & =F\left(\begin{array}{cc}
A z & -1 \\
1 & 0
\end{array}\right)=F\left(\left(\begin{array}{ll}
a & b \\
c & d
\end{array}\right)\left(\begin{array}{cc}
z & -1 \\
1 & 0
\end{array}\right)\left(\begin{array}{cc}
\operatorname{det}(A) \mathrm{j}(A, z)^{-1} & c \\
0 & \mathrm{j}(A, z)
\end{array}\right)\right) \\
& =\mathrm{j}(A, z)^{n} \mathrm{j}(A, z)^{m-n} \sum_{i=0}^{n}\left(\begin{array}{l}
n \\
i
\end{array}\right) c^{i} \mathrm{j}(A, z)^{-i} f_{i}(x)=\sum_{i=0}^{n}\left(\begin{array}{c}
n \\
i
\end{array}\right) c^{i} \mathrm{j}(A, z)^{m-i} f_{i}(x) .
\end{aligned}
$$

In the second equality we have used the facts that $F$ is $\mathrm{SL}(2, \mathbb{Z})$ invariant and it satisfies the property (19). We have finished the proof of our proposition.

We denote by $\check{M}_{m}^{n}$ the set of holomorphic functions on $\mathcal{P}$ which are left $\operatorname{SL}(2, \mathbb{Z})$ invariant and satisfy (19) for some holomorphic functions $F_{i}$ on $\mathcal{P}$ such that $F_{i}$ 's restricted to $\tilde{\mathbb{H}}$ have finite growths at infinity. For the determinant function det $: \mathcal{P} \rightarrow \mathbb{C}$ we have:

$$
\operatorname{det} \in \check{M}_{0}^{1},(\operatorname{det})^{i} \check{M}_{m}^{n} \subset M_{m}^{\check{n}+i}, i \in \mathbb{N}_{0} .
$$

In a similar way as in Proposition 3, one can prove that:

$$
\check{M}_{m}^{n} \check{M_{m^{\prime}}^{n^{\prime}}} \subset M_{m+m^{\prime}}^{n^{\prime}+n^{\prime}}, \check{M}_{m}^{n}+(\operatorname{det})^{n^{\prime}-n} \check{M}_{m}^{n^{\prime}}=\check{M_{m}^{n^{\prime}}} m, m^{\prime} \in \mathbb{Z}, n, n^{\prime} \in \mathbb{N}_{0}, n \leq n^{\prime} .
$$

We have an isomorphism $\check{M}_{m}^{n} \rightarrow M_{m}^{n},\left.F \mapsto F\right|_{\tilde{\mathbb{H}}}$ whose inverse is given by $\phi$ in Proposition 6. For a classical modular form $f: \mathbb{H} \rightarrow \mathbb{C}$ of weight $m$ the associated $F=\phi(f)$ is

$$
F(x)=x_{3}^{m} f\left(\frac{x_{1}}{x_{3}}\right) \in \check{M}_{m}^{0} .
$$




\subsection{Proof of Proposition 5}

We define

$$
\check{T}_{p}: \check{M}_{m}^{n} \rightarrow \check{M}_{m}^{n}, \check{T}_{p} F(x)=p^{m-2 n-1} \sum_{k=1}^{s} F\left(A_{i} x\right) .
$$

This function has trivially its image in $\check{M}_{m}^{n}$. We calculate the corresponding function in $M_{m}^{n}$ : The term $T_{p} f(z)$ is equal to:

$$
\begin{aligned}
& =p^{m-2 n-1} \sum_{k=1}^{s} F\left(A_{k}\left(\begin{array}{cc}
z & -1 \\
1 & 0
\end{array}\right)\right) \\
& =p^{m-2 n-1} \sum_{k=1}^{s} F\left(\left(\begin{array}{cc}
A_{k} z & -1 \\
1 & 0
\end{array}\right)\left(\begin{array}{cc}
\mathrm{j}\left(A_{k}, z\right) & -c \\
0 & p \cdot \mathrm{j}\left(A_{k}^{-1}, A_{k} z\right)
\end{array}\right)\right) \\
& =p^{m-2 n-1} \sum_{k=1}^{s}\left(p \cdot \mathrm{j}\left(A_{k}^{-1}, A_{k} z\right)\right)^{n}\left(\mathrm{j}\left(A_{k}, z\right)\right)^{n-m} \sum_{i=0}^{n}\left(\begin{array}{c}
n \\
i
\end{array}\right)\left(-c p^{-1}\right)^{i} \mathrm{j}\left(A_{k}^{-1}, A_{k} z\right)^{-i} f_{i}\left(A_{k} z\right) \\
& =\sum_{k=1}^{s} f \|_{m} A_{k}
\end{aligned}
$$

where $A_{k}=\left(\begin{array}{ll}a & b \\ c & d\end{array}\right)$. This proves Proposition 5

\subsection{Some non-holomorphic functions on the period domain}

We now define some functions that will be used in the proof of Theorem 2. Their relation with the functions of Theorem 2 will be explained in the next sections, where we have introduced the period map.

On the complex manifold $\mathcal{P}$ we have the following left $\mathrm{SL}(2, \mathbb{Z})$ invariant analytic functions:

$$
B_{1}:=\operatorname{Im}\left(x_{1} \overline{x_{3}}\right), B_{2}:=\operatorname{Im}\left(x_{2} \overline{x_{4}}\right), B_{3}:=x_{1} \overline{x_{4}}-x_{2} \overline{x_{3}} .
$$

They define analytic functions on $\mathcal{L}$ which we denote them by the same letter. They satisfy

$$
\begin{gathered}
\left.B_{1}\right|_{\tilde{\mathbb{H}}}(z)=\operatorname{Im}(z), B_{1}(x g)=B_{1}(x)\left|k_{1}\right|^{2} \\
\left.B_{2}\right|_{\tilde{\mathbb{H}}}(z)=0, B_{2}(x g)=B_{1}(x)\left|k_{3}\right|^{2}+B_{2}(x)\left|k_{2}\right|^{2}+\operatorname{Im}\left(B_{3}(x) k_{3} \overline{k_{2}}\right) \\
\left.B_{3}\right|_{\tilde{\mathbb{H}}}(z)=1, B_{3}(x g)=B_{3}(x) k_{1} \overline{k_{2}}+2 \sqrt{-1} k_{1} \overline{k_{3}} B_{1}(x) .
\end{gathered}
$$

By the equality (20) one can easily see that every point in $\mathcal{P}$ can be mapped to a point of $\tilde{\mathbb{H}}$ by an action of a unique element of $G_{0}$. This implies that the $\operatorname{SL}(2, \mathbb{Z})$ invariant functions $B_{i}, i=1,2,3$, with the above properties are unique. 


\section{Families of elliptic curves and the Gauss-Manin connec- tion}

In this section we consider the following family of elliptic curves

$$
E: y^{2}-4 t_{0}\left(x-t_{1}\right)^{3}+t_{2}\left(x-t_{1}\right)+t_{3}=0
$$

and its specialization $E_{t}$ over a regular point $t \in T:=\mathbb{C}^{4} \backslash\{\Delta=0\}$, where $\Delta=t_{0}\left(27 t_{0} t_{3}^{2}-\right.$ $\left.t_{2}^{3}\right)$ is the discriminant of $E$. We have a proper smooth morphism $E \rightarrow T$ defined over $\mathbb{C}$. The family $E$ with $t_{0}=1$ and $t_{1}=0$ is the classical Weierstrass family of elliptic curves and the material of the sections 3.1, 3.2 and 3.3 for such a family is well-known (see for instance 9] Appendix 1, 4] and [14]). The discussions related to the full family (28) are slight modifications of the classical ones.

\subsection{Gauss-Manin connection}

The algebraic definition of the Gauss-Manin connection is made by N. M. Katz, T. Oda 1968 and P. Deligne 1971. Its computational aspects are discussed in [11. Let us introduce the basic notations for the Gauss-Manin connection of the family $E$.

The Gauss-Manin connection on the cohomology bundle $\mathcal{H}_{\mathrm{dR}}^{1}(E / T)$ is a $\mathbb{C}$-linear map:

$$
\nabla: \mathcal{H}_{\mathrm{dR}}^{1}(E / T) \rightarrow \Omega_{T}^{1} \otimes_{\mathcal{O}_{T}} \mathcal{H}_{\mathrm{dR}}^{1}(E / T),
$$

where $\Omega_{T}^{1}$ is the sheaf of differential 1-forms on $T$. It satisfies $\nabla(f e)=d f \otimes e+f \nabla(e), f$ (resp. e) being a section of $\mathcal{O}_{T}\left(\right.$ resp. $\left.\mathcal{H}_{\mathrm{dR}}^{1}(E / T)\right)$. The set

$$
H:=H_{\mathrm{dR}}^{1}(E / T)
$$

of global sections of $\mathcal{H}_{\mathrm{dR}}^{1}(E / T)$ is a $\mathbb{C}\left[t, \frac{1}{\Delta}\right]$-module generated freely by the classical differential forms $\frac{d x}{y}, \frac{x d x}{y}$. For $\omega=\left(\frac{d x}{y}, \frac{x d x}{y}\right)^{\operatorname{tr}}$, the Gauss-Manin connection can be written in the following way:

$$
\nabla \omega=A \otimes \omega, A=\frac{1}{\Delta}\left(\sum_{i=0}^{3} A_{i} d t_{i}\right), A_{i} \in \operatorname{Mat}(2, \mathbb{C}[t]) .
$$

A simple calculation shows that:

(30)

$$
\begin{aligned}
& A_{0}=\left(\begin{array}{cc}
3 / 2 t_{0} t_{1} t_{2} t_{3}-9 t_{0} t_{3}^{2}+1 / 4 t_{2}^{3} & -3 / 2 t_{0} t_{2} t_{3} \\
3 / 2 t_{0} t_{1}^{2} t_{2} t_{3}+9 t_{0} t_{1} t_{3}^{2}-1 / 2 t_{1} t_{2}^{3}+1 / 8 t_{2}^{2} t_{3} & -3 / 2 t_{0} t_{1} t_{2} t_{3}-18 t_{0} t_{3}^{2}+3 / 4 t_{2}^{3}
\end{array}\right) \\
& A_{1}=\left(\begin{array}{cc}
0 & 0 \\
27 t_{0}^{2} t_{3}^{2}-t_{0} t_{2}^{3} & 0
\end{array}\right) \\
& A_{2}=\left(\begin{array}{cc}
-9 / 2 t_{0}^{2} t_{1} t_{3}+1 / 4 t_{0} t_{2}^{2} & 9 / 2 t_{0}^{2} t_{3} \\
-9 / 2 t_{0}^{2} t_{1}^{2} t_{3}+1 / 2 t_{0} t_{1} t_{2}^{2}-3 / 8 t_{0} t_{2} t_{3} & 9 / 2 t_{0}^{2} t_{1} t_{3}-1 / 4 t_{0} t_{2}^{2}
\end{array}\right) \\
& A_{3}=\left(\begin{array}{cc}
3 t_{0}^{2} t_{1} t_{2}-9 / 2 t_{0}^{2} t_{3} & -3 t_{0}^{2} t_{2} \\
3 t_{0}^{2} t_{1}^{2} t_{2}-9 t_{0}^{2} t_{1} t_{3}+1 / 4 t_{0} t_{2}^{2} & -3 t_{0}^{2} t_{1} t_{2}+9 / 2 t_{0}^{2} t_{3}
\end{array}\right) .
\end{aligned}
$$

(See [1] for the procedures which calculate all matrices above). Let $U$ be an small open set in $U$ and $\left\{\delta_{t}\right\}_{t \in U}, \delta_{t} \in H_{1}\left(E_{t}, \mathbb{Z}\right)$ be a continuous family of topological one dimensional cycles. The main property of the Gauss-Manin connection is:

$$
d\left(\int_{\delta_{t}} \eta\right)=\sum \alpha_{i} \int_{\delta_{t}} \beta_{i}, \nabla \eta=\sum_{i} \alpha_{i} \otimes \beta_{i}, \alpha_{i} \in H^{0}\left(T, \Omega_{T}^{1}\right), \eta, \beta_{i} \in H .
$$




\subsection{Period map}

The period map associated to the basis $\omega:=\left(\frac{d x}{y}, \frac{x d x}{y}\right)^{\operatorname{tr}}$ is given by:

$$
\mathrm{pm}: T \rightarrow \mathrm{SL}(2, \mathbb{Z}) \backslash \mathcal{P}, t \mapsto\left[\frac{1}{\sqrt{2 \pi i}}\left(\begin{array}{ll}
\int_{\delta_{1}} \omega_{1} & \int_{\delta_{1}} \omega_{2} \\
\int_{\delta_{2}} \omega_{1} & \int_{\delta_{2}} \omega_{2}
\end{array}\right)\right]
$$

It is well-defined and holomorphic. Here $\sqrt{i}=e^{\frac{2 \pi i}{4}}$ and $\left(\delta_{1}, \delta_{2}\right)$ is a basis of the $\mathbb{Z}$-module $H_{1}\left(E_{t}, \mathbb{Z}\right)$ such that the intersection matrix in this basis is $\left(\begin{array}{cc}0 & 1 \\ -1 & 0\end{array}\right)$. It follows from (31) that pm satisfies the differential equation:

$$
d(\mathrm{pm})(t)=\mathrm{pm}(t) \cdot A^{\mathrm{tr}}, t \in T
$$

where $d$ is the differential map.

\subsection{The Action of an algebraic group}

We consider the family of elliptic curves (28). It can be checked easily that (9) is an action of $G_{0}$ on $\mathbb{C}^{4}$ (this can be also verified from the proof of the proposition bellow). It is also easy to verify that $\mathbb{C}^{4} / G_{0}$ is isomorphic to $\mathbb{P}^{1}$ through the map

$$
s: \mathbb{C}^{4} / G_{0} \rightarrow \mathbb{P}^{1}, t \rightarrow\left[t_{2}^{3}: 27 t_{0} t_{3}^{2}-t_{2}^{3}\right]
$$

and so

$$
j(t):=\frac{t_{2}^{3}}{27 t_{0} t_{3}^{2}-t_{2}^{3}}
$$

is $G_{0}$-invariant and gives an isomorphy between $T / G_{0}$ and $\mathbb{C}$.

Proposition 7. The period $\mathrm{pm}$ associated to the basis $\omega$ is a biholomorphism and

$$
\operatorname{pm}(t \bullet g)=\operatorname{pm}(t) \cdot g, t \in \mathbb{C}^{4}, g \in G_{0} .
$$

Proof. We first prove (35). Let

$$
\alpha: \mathbb{C}^{2} \rightarrow \mathbb{C}^{2},(x, y) \mapsto\left(k_{2}^{-1} k_{1} x-k_{3} k_{2}^{-1}, k_{2}^{-1} k_{1}^{2} y\right) .
$$

Then

$$
\begin{gathered}
k_{2}^{2} k_{1}^{-4} \alpha^{-1}(f)=y^{2}-4 t_{0} k_{2}^{2} k_{1}^{-4}\left(k_{2}^{-1} k_{1} x-k_{3} k_{2}^{-1}-t_{1}\right)^{3}+t_{2} k_{2}^{2} k_{1}^{-4}\left(k_{2}^{-1} k_{1} x-k_{3} k_{2}^{-1}-t_{1}\right)+t_{3} k_{2}^{2} k_{1}^{-4} \\
y^{2}-4 t_{0} k_{1}^{-1} k_{2}^{-1}\left(x-\left(t_{1} k_{2} k_{1}^{-1}+k_{3} k_{1}^{-1}\right)\right)^{3}+t_{2} k_{1}^{-3} k_{2}\left(x-\left(t_{1} k_{2} k_{1}^{-1}+k_{3} k_{1}^{-1}\right)\right)+t_{3} k_{1}^{-4} k_{2}^{2}
\end{gathered}
$$

This implies that $\alpha$ induces an isomorphism of elliptic curves

$$
\alpha: E_{t \bullet g} \rightarrow E_{t} .
$$

Now

$$
\alpha^{-1} \omega=\left(\begin{array}{cc}
k_{1}^{-1} & 0 \\
-k_{3} k_{2}^{-1} k_{1}^{-1} & k_{2}^{-1}
\end{array}\right) \omega=\left(\begin{array}{cc}
k_{1} & 0 \\
k_{3} & k_{2}
\end{array}\right)^{-1} \omega
$$

and so

$$
\operatorname{pm}(t)=\operatorname{pm}(t \bullet g) \cdot g^{-1}
$$


which proves 35 .

Let $B$ be the $4 \times 4$ matrix whose $i$-th row, $i=1,2, \ldots, 4$, constitutes of the first and second rows of $A_{i-1}$. A simple calculation shows that

$$
\operatorname{det}(B)=\frac{3}{4} t_{0} \Delta^{3}
$$

and so the period map $\mathrm{pm}$ is regular at each point $t \in T$. Therefore, it is locally a biholomorphism.

The period map pm induces a local biholomorphic map pm : $T / G_{0} \rightarrow \mathrm{SL}(2, \mathbb{Z}) \backslash \mathbb{H} \cong$ $\mathbb{C}$ and so we have the local biholomorphism $\mathrm{pm}^{\circ} \circ j^{-1}: \mathbb{C} \rightarrow \mathbb{C}$. One can compactify $\operatorname{SL}(2, \mathbb{Z}) \backslash \mathbb{H}$ by adding the cusp $\operatorname{SL}(2, \mathbb{Z}) / \mathbb{Q}=\{c\}$ (see 10 ) and the map $\mathrm{pm} \circ j^{-1}$ is continuous at $v$ sending $v$ to $c$, where $v$ is the point induced by $27 t_{0} t_{3}^{2}-t_{2}^{3}=0$ in $\mathbb{C}^{4} / G_{0}$. Using Picard's Great Theorem we conclude that $j^{-1} \circ \overline{p m}$ is a biholomorphism and so pm is a biholomorphism.

\subsection{The inverse of the period map}

We denote by

$$
F=\left(F_{0}, F_{1}, F_{2}, F_{3}\right): \mathcal{P} \rightarrow T
$$

the composition of the quotient map $\mathcal{P} \rightarrow \mathrm{SL}(2, \mathbb{Z}) \backslash \mathcal{P}$ and the inverse of the period map.

Proposition 8. The following is true:

1. $F_{0}(x)=\operatorname{det}(x)^{-1}$.

2. For $i=2,3$

$$
F_{i}=\operatorname{det}(x)^{1-i} \check{g}_{i} \in \check{M}_{2 i}^{0}
$$

where $g_{i}$ is the Eisenstein series (1).

3. $F_{1}=\check{g_{1}} \in \check{M}_{2}^{1}$.

Proof. Taking $F$ of (35) we have

$$
\begin{gathered}
F_{0}(x g)=F_{0}(x) k_{1}^{-1} k_{2}^{-1}, \\
F_{1}(x g)=F_{1}(x) k_{1}^{-1} k_{2}+k_{3} k_{1}^{-1}, \\
F_{2}(x g)=F_{2}(x) k_{1}^{-3} k_{2}, F_{3}(x g)=F_{3}(x) k_{1}^{-4} k_{2}^{2}, \forall x \in \mathcal{L}, g \in G_{0} .
\end{gathered}
$$

By the Legendre's theorem $\operatorname{det}(x)$ is equal to one on $V:=\operatorname{pm}(1 \times 0 \times \mathbb{C} \times \mathbb{C})$ and so the same is true for $F_{0} \operatorname{det}(x)$. But the last function is invariant under the action of $G_{0}$ and so it is the constant function 1 . This proves the first item. Let $G_{i}=F_{i} \operatorname{det}(x)^{i-1}, i=1,2,3$. The equalities (36) imply that $G_{i}, i=2,3$ do not depend on $x_{2}, x_{4}$. Now the map $\left(t_{2}, t_{3}\right) \rightarrow$ $\pi \circ \mathrm{pm}\left(1,0, t_{2}, t_{3}\right)$, where $\pi$ is the projection on the $x_{1}, x_{3}$ coordinates, is the classical period map (see for instance the appendix of [9]) and this implies that $G_{i}=\check{g}_{i}, i=2,3$. Note that in our definition of the period map the factor $\frac{1}{\sqrt{2 \pi i}}$ appears. In particular $F_{i}, i=2,3$ have finite growths at infinity. The fact that $F_{1}$ has a finite growth at infinity follows form the Ramanujan relations (18) and the equality $\frac{d}{d z}=2 \pi i q \frac{d}{d q}$. Since $G_{1} \in \check{M}_{2}^{1}, \check{M}_{2}^{1}$ is a one dimensional space, both $g_{1}, G_{1}$ satisfy (36) and $M_{2}^{0}=\{0\}$, we have $G_{1}=g_{1}$. 


\subsection{Ramanujan relations}

We proved in Proposition 7 that the period map pm associated to $\omega$ is a biholomorphism. According to (32), the inverse $F$ of pm satisfies the differential equation

$$
x \cdot A(F(x))^{\mathrm{tr}}=I .
$$

We consider pm as a map sending the vector $\left(t_{0}, t_{1}, t_{2}, t_{3}\right)$ to $\left(x_{1}, x_{2}, x_{3}, x_{4}\right)$. Its derivative at $t$ is a $4 \times 4$ matrix whose $i$-th column constitutes of the first and second row of $\frac{1}{\Delta} x A_{i}^{\text {tr }}$. We use (30) to derive the equality

$$
\begin{aligned}
& (d F)_{x}=(d \mathrm{pm})_{t}^{-1}= \\
& \operatorname{det}(x)^{-1}\left(\begin{array}{cccc}
-F_{0} x_{4} & F_{0} x_{3} & & -F_{0} x_{2} \\
\frac{1}{12 F_{0}}\left(12 F_{0} F_{1}^{2} x_{3}-12 F_{0} F_{1} x_{4}-F_{2} x_{3}\right) & -F_{1} x_{3}+x_{4} & \frac{1}{12 F_{0}}\left(-12 F_{0} F_{1}^{2} x_{1}+12 F_{0} F_{1} x_{2}+F_{2} x_{1}\right) & F_{1} F_{1}-x_{2} \\
\frac{1}{4 F_{1}}\left(18 F_{2} x_{3}-3 F_{2} F_{1} F_{3} x_{3}-12 F_{0}-6 F_{3} x_{3} x_{3}-F_{2}^{2} x_{3}\right) & -2 F_{2} x_{3} x_{3} & \frac{1}{3 F_{0}}\left(-18 F_{0} F_{2} F_{1}+3 F_{3} x_{1}+12 F_{2} x_{2}+6 F_{0} F_{3} F_{3} x_{1}+F_{2}^{2} x_{1}\right) & F_{2} x_{1} \\
\hline 3 F_{3} x_{1}
\end{array}\right) .
\end{aligned}
$$

For $g_{i}:=\left.F_{i}\right|_{\tilde{H}}$ the first column of the above equality gives us the Ramanujan relations (18).

\subsection{The family $y^{2}-4 t_{0} x^{3}+t_{1} x^{2}+t_{2} x+t_{3}$}

The family (28) can be rewritten in the form

$$
y^{2}-4 t_{0} x^{3}+12 t_{0} t_{1} x^{2}+\left(-12 t_{0} t_{1}^{2}+t_{2}\right) x+\left(4 t_{0} t_{1}^{3}-t_{2} t_{1}+t_{3}\right)=0 .
$$

The mapping

$$
\alpha: \mathbb{C}^{4} \rightarrow \mathbb{C}^{4}, t \mapsto\left(t_{0}, 12 t_{0} t_{1},-12 t_{0} t_{1}^{2}+t_{2}, 4 t_{0} t_{1}^{3}-t_{2} t_{1}+t_{3}\right)
$$

is an isomorphism and so we can restate Proposition 7 for the family

$$
E_{t}: y^{2}-4 t_{0} x^{3}+t_{1} x^{2}+t_{2} x+t_{3}=0
$$

The inverse of the period map in this case is given by $G=\left(G_{0}, G_{1}, G_{2}, G_{3}\right)$ with

$$
G_{0}=F_{0}, G_{1}=12 F_{0} F_{1}, G_{2}=-12 F_{0} F_{1}^{2}+F_{2}, G_{3}=4 F_{0} F_{1}^{3}-F_{2} F_{1}+F_{3} .
$$

In this case the singular fibers are parameterized by the zeros of

$$
\Delta:=t_{0}\left(432 t_{0}^{2} t_{3}^{2}+72 t_{0} t_{1} t_{2} t_{3}-16 t_{0} t_{2}^{3}+4 t_{1}^{3} t_{3}-t_{1}^{2} t_{2}^{2}\right) .
$$

The Ramanujan relations take the simpler form:

$$
\left\{\begin{array}{l}
\dot{t_{1}}=-t_{2} \\
\dot{t_{2}}=-6 t_{3} \\
\dot{t_{3}}=t_{1} t_{3}-\frac{1}{4} t_{2}^{2}
\end{array},\right.
$$

where

$$
\left(t_{1}, t_{2}, t_{3}\right):=\left(12 g_{1},-12 g_{1}^{2}+g_{2}, 4 g_{1}^{3}-g_{2} g_{1}+g_{3}\right)
$$

\section{Proofs}

Now we are in a position to prove the theorems announced in the Introduction. 


\subsection{Proof of Theorem 1}

It is enough to prove that

$$
\check{M}:=\sum_{m, i \in \mathbb{Z}, n \in \mathbb{N}_{0}} F_{0}^{i} \check{M}_{m}^{n}
$$

as a $\mathbb{C}\left(F_{0}\right)$-algebra is freely generated by $F_{i}, i=1,2,3$ and every $F \in \check{M}_{m}^{n}$ can be written as a homogeneous polynomials of degree $m$ in the graded ring $\mathbb{C}\left(F_{0}\right)\left[F_{1}, F_{2}, F_{3}\right], \operatorname{deg}\left(F_{i}\right)=$ $2 i, i=1,2,3$ and of degree $2 n$ in $F_{1}$. Since $F_{0}=\left.(\text { det })^{-1}\right|_{\tilde{\mathbb{H}}}=1$ and $\left.F_{i}\right|_{\tilde{\mathbb{H}}}=\tilde{g}_{i}, i=1,2,3$, this will imply Theorem 1 .

Since the period map is a biholomorphism (Proposition [7) and the pull-back of $F_{i}, i=$ $0,1,2,3$ by the period map is $t_{i}$ and $t_{1}, t_{2}, t_{3}$ are algebraically independent over $\mathbb{C}\left(t_{0}\right)$, we conclude that $F_{i}, i=1,2,3$ are algebraically independent over $\mathbb{C}\left(F_{0}\right)$.

Again we use that fact that the period map is a biholomorphism and conclude that for $\tilde{F} \in \check{M}_{m}^{n}$ and its associated functions $\tilde{F}_{i} \in M_{m-2 i}^{\check{n}-i}$, there exist holomorphic functions $p_{i}: T \rightarrow \mathbb{C}, i=0,1, \ldots, n, p_{0}:=p$ such that $\tilde{F}_{i}=p_{i}\left(F_{0}, F_{1}, F_{2}, F_{3}\right), i=0,1,2, \ldots, n$. The property (19) of $\tilde{F}$ and (35) imply that:

$$
p(t \bullet g)=k_{2}^{n} k_{1}^{n-m} \sum_{i=0}^{n}\left(\begin{array}{c}
n \\
i
\end{array}\right) k_{3}^{i} k_{2}^{-i} p_{i}(t), \forall g \in G_{0}, t \in T .
$$

Take $g=\left(\begin{array}{cc}1 & t_{1} \\ 0 & 1\end{array}\right)$ and $t=\left(t_{0}, 0, t_{1}, t_{3}\right)$. Then

$$
p\left(t_{0}, t_{1}, t_{2}, t_{3}\right)=\sum_{i=0}^{n}\left(\begin{array}{l}
n \\
i
\end{array}\right) t_{1}^{i} p_{i}\left(t_{0}, 0, t_{2}, t_{3}\right) .
$$

This implies that $p$ is a polynomial of degree at most $2 n$ in the variable $t_{1}\left(\operatorname{deg}\left(t_{1}\right)=2\right)$. In (38) we take $g=\left(\begin{array}{cc}k_{1} & 0 \\ 0 & t_{0} k_{1}^{-1}\end{array}\right)$ and obtain

$$
p\left(1, t_{1} t_{0} k_{1}^{-2}, t_{2} t_{0} k_{1}^{-4}, t_{3} t_{0}^{2} k_{1}^{-6}\right)=t_{0}^{n} k_{1}^{-m} p(t) .
$$

We substitute (39) in (40) and consider the equalities obtained by the coefficients of $t_{1}^{i}$. We get

$$
p_{i}\left(1,0, t_{2} t_{0} k_{1}^{-4}, t_{3} t_{0}^{2} k_{1}^{-6}\right)=t_{0}^{n-i} k_{1}^{-m+2 i} p_{i}\left(t_{0}, 0, t_{2}, t_{3}\right), i=0,1,2, \ldots, n .
$$

We take $t_{0}=1$ and conclude that $p_{i}\left(1,0, F_{2}, F_{3}\right) \in M_{m-2 i}^{0^{\circ}}$. Since every modular form of weight $m-2 i$ can be written as a homogeneous polynomial of degree $m-2 i$ in $\mathbb{C}\left[g_{2}, g_{3}\right], \operatorname{deg}\left(g_{2}\right)=4, \operatorname{deg}\left(g_{3}\right)=6, p_{i}\left(1,0, F_{2}, F_{3}\right)$ can be written as a homogeneous polynomial of degree $m-2 i$ in $\mathbb{C}\left[F_{2}, F_{3}\right], \operatorname{deg}\left(F_{2}\right)=4, \operatorname{deg}\left(F_{3}\right)=6$. In (41) we put $k_{1}=1$ and conclude that $p_{i}\left(t_{0}, 0, t_{2}, t_{3}\right)=t_{0}^{i-n} p_{i}\left(1,0, t_{2} t_{0}, t_{3} t_{0}^{2}\right)$ is a homogeneous polynomial of degree $m-2 i$ in $\mathbb{C}\left(t_{0}\right)\left[t_{2}, t_{3}\right], \operatorname{deg}\left(t_{2}\right)=4, \operatorname{deg}\left(t_{3}\right)=6$.

\subsection{Proof of Theorem 2}

In $\$ 2.6$ we described some analytic functions $B_{i}, i=1,2,3$, on $\mathcal{L}$ which have the compatibility properties (25),(26) and (27) with the action of $G_{0}$ on $\mathcal{L}$. We use Proposition 7 and 
transfer them to the world of coefficients $T$. We obtain analytic functions $B_{1}, B_{2}: T \rightarrow \mathbb{R}$ and $B_{3}: T \rightarrow \mathbb{C}$ which satisfy:

$$
\begin{gathered}
B_{1} \circ g(z)=\operatorname{Im}(z), B_{1}(t \bullet g)=B_{1}(t)\left|k_{1}\right|^{2} \\
B_{2} \circ g(z)=0, B_{2}(t \bullet g)=B_{1}(t)\left|k_{3}\right|^{2}+B_{2}(t)\left|k_{2}\right|^{2}+\operatorname{Im}\left(B_{3}(t) k_{3} \overline{k_{2}}\right) \\
B_{3} \bullet g(z)=1, B_{3}(t \bullet g)=B_{3}(t) k_{1} \overline{k_{2}}+2 \sqrt{-1} k_{1} \overline{k_{3}} B_{1}(t) .
\end{gathered}
$$

for $t \in T, z \in \mathbb{H}$ and $g \in G_{0}$, where $g: \mathbb{H} \rightarrow T, g(z)=\left(1, g_{1}(z), g_{2}(z), g_{3}(z)\right.$ ) (for the sake of simplicity we have used the same letters to name these functions). In (42) we put $g=\left(\begin{array}{cc}1 & -t_{1} \\ 0 & 1\end{array}\right)$ and obtain $B_{1}\left(t_{0}, 0, t_{2}, t_{3}\right)=B_{1}\left(t_{0}, t_{1}, t_{2}, t_{3}\right)$ which means that $B_{1}$ does not depend on $t_{1}$. Now in (42) we put $t_{0}=1$ and $g=\left(\begin{array}{cc}k & 0 \\ 0 & k^{-1}\end{array}\right)$ and obtain (4). In (43) and (44) we put $t_{0}=1$ and $g=\left(\begin{array}{cc}k & k^{\prime} \\ 0 & k^{-1}\end{array}\right)$ and obtain the equalities (5) and (6). The uniqueness of $B_{1}, B_{2}$ and $B_{3}$ in Theorem $[2$ follows form uniqueness of the same functions in the period domain.

The proof of the last part of the theorem is as follows: If $B_{2}(x):=\operatorname{Im}\left(x_{2} \bar{x}_{4}\right)=0$ for some $x \in \mathcal{P}$ with $\operatorname{det}(x)=1$ then $x=\left(\begin{array}{cc}x_{1} & x_{4} r \\ x_{3} & x_{4}\end{array}\right)$ for some $r \in \mathbb{R}$ and $x_{4}\left(x_{1}-r x_{3}\right)=1$. Then

$$
B_{3}(x)=\overline{x_{4}}\left(x_{1}-r x_{3}\right)=\frac{\overline{x_{4}}}{x_{4}} .
$$

which implies that $\left|B_{3}(x)\right|=1$. In the coefficient space $\operatorname{det}(x)=1$ corresponds to $t_{0}=1$ and so we get the last statement of Theorem 2 .

One can say something more about $B_{1}$ : The function $B_{1} \cdot|\Delta|^{\frac{1}{6}}$ is $G_{0}$ invariant and so there is an analytic function $b_{1}: \mathbb{C} \rightarrow \mathbb{R}$ such that

$$
B_{1}(t)=\frac{b_{1}(j(t))}{|\Delta(t)|^{\frac{1}{6}}}
$$

Taking this equality to the period domain and restricting it to $\tilde{\mathbb{H}}$, we get

$$
\operatorname{Im}(z)=\frac{b_{2}(j(z))}{|\Delta(z)|^{\frac{1}{6}}}
$$

where the above $j$ and $\Delta$ are the ones on $\$ 2.2$

\subsection{Proof of Theorem 3}

Let $k$ be an algebraically closed field of charachteristic 0 , for instance take $k=\overline{\mathbb{Q}}$. By a variety over $k$ we mean the set of its $k$-rational points. We redefine:

$$
G_{0}:=\left\{\left(\begin{array}{cc}
k_{1} & k_{3} \\
0 & k_{2}
\end{array}\right) \mid k_{3} \in k, k_{1}, k_{2} \in k^{*}\right\}, T:=k^{4} \backslash\left\{t \in k^{4} \mid t_{0}\left(27 t_{0} t_{3}^{2}-t_{2}^{3}\right)=0\right\} .
$$


Proposition 9. The quasi affine variety $T$ is the moduli of $\left(F,\left[\omega_{1}\right],\left[\omega_{2}\right]\right)$ 's, where $F$ is an elliptic curve defined over $k, \omega_{1}$ is a differential form of the first kind on $F$ and $\left(\left[\omega_{1}\right],\left[\omega_{2}\right]\right)$ is a basis of $H_{\mathrm{dR}}^{1}(F)$.

Proof. For simplicity we do not write more [.] for differential forms. The $j$ invariant (34) classifies the ellipric curves over $k$ (see [5] Theorem 4.1). Therefore, for a given elliptic curve $F / k$ we can find parameter a $t \in k^{4}$ such that $F \cong E_{t}$ over $k$. Under this isomorphism we write

$$
\left(\begin{array}{l}
\omega_{1} \\
\omega_{2}
\end{array}\right)=g^{\operatorname{tr}}\left(\begin{array}{c}
\frac{d x}{y} \\
\frac{x d x}{y}
\end{array}\right), \text { in } H_{\mathrm{dR}}^{1}\left(E_{t}\right)
$$

for some $g \in G_{0}$, where $\omega_{1}, \omega_{2}$ are as in the proposition. Now, the triple $\left(F, \omega_{1}, \omega_{2}\right)$ is isomorphic to $\left(E_{t \bullet g}, \frac{d x}{y}, \frac{x d x}{y}\right)$. Since $j: \mathbb{C}^{4} / G_{0} \rightarrow \mathbb{C}$ is an isomorphism, every triple $\left(F, \omega_{1}, \omega_{2}\right)$ is represented exactly by one parameter $t \in T$.

Let us take $k=\overline{\mathbb{Q}}$. By Proposition 9 the hypothesis of Theorem 3 gives us a parameter $t \in T$ such that $\int_{\delta} \frac{x d x}{y}=0$, for some $\delta \in H_{1}\left(E_{t}, \mathbb{Z}\right)$. We can assume that $\delta$ is not a multiple of another cycle in $H_{1}\left(E_{t}, \mathbb{Z}\right)$ and so we can find another cycle $\delta^{\prime}$ in $H_{1}\left(E_{t}, \mathbb{Z}\right)$ such that $\left\langle\delta, \delta^{\prime}\right\rangle=1$. The corresponding period matrix $x$ of $E_{t}$ in $\left(\delta^{\prime}, \delta\right)$ has zero $x_{4}$-coordinate and so the numbers

$$
t_{0}=\operatorname{det}(x)^{-1}, t_{i}=F_{i}(x)=\operatorname{det}(x)^{1-i} x_{3}^{-2 i} g_{i}\left(\frac{x_{1}}{x_{3}}\right), i=2,3, t_{1}=F_{1}(x)=\operatorname{det}(x) x_{3}^{-2} g_{1}\left(\frac{x_{1}}{x_{3}}\right)
$$

all are in $\overline{\mathbb{Q}}$. Here we have used Proposition 8 and $x_{4}=0$. Now, for $z=\frac{x_{1}}{x_{3}} \in \mathbb{H}$ we have

$$
\frac{g_{3}}{g_{1}^{3}}(z), \frac{g_{2}}{g_{1}^{2}}(z), \frac{g_{3}^{2}}{g_{2}^{3}}(z) \in \overline{\mathbb{Q}} \text {. }
$$

This is in contradiction with

Theorem (Nesterenko 1996, 13]) For any $z \in \mathbb{H}$, the set

$$
e^{2 \pi i z}, \frac{g_{1}(z)}{a_{1}}, \frac{g_{2}(z)}{a_{2}}, \frac{g_{3}(z)}{a_{3}}
$$

contains at least three algebraically independent numbers over $\mathbb{Q}$.

A direct corollary of Theorem 3 is that the multi-valued function

$$
I(t)=\frac{\int_{\delta_{t}} \frac{x d x}{y}}{\int_{\delta_{t}} \frac{d x}{y}}
$$

defined in $T$ never takes algebraic values for algebraic $t$.

\subsection{Other topics}

The literature of modular forms and its applications in number theory is huge. The first question which naturally arises at this point is as follows: Which part of the theory of modular forms can be generalized to the context of differential modular forms and which arithmetic properties can one expect to find? Since I am not expert in this area, I just mention some subjects which could fit well into this section.

One may ask for the Eichler-Manin-Shimura theory of periods for cusp forms (see 6 and its references) in the context of differential modular forms. Note that the notion 
"period" in this theory, as far as I know, has nothing to do with the notion of a period in this article. The notion of period appears there because classical modular forms can be interpreted as sections of a tensor product of the cotangent bundle of a moduli curve and hence a differential multi form, which can be integrated over some path in the moduli curve (see [15]). The differential modular forms are no longer interpreted as sections of line bundles and this makes the situation more difficult. Lewis type equations attached to differential modular forms will be also of interest (see [6]).

Another theory which could be developed for differential modular forms is AtkinLehner theory of old and new modular forms (see the references in [6]). This seems to me to be a quite accessible theory. The $L$-functions attached to differential modular forms through their Fourier expansion and the extension of the Rankin-Cohen bracket to differential modular forms may be also of interest.

\section{References}

[1] T. M. Apostol. Modular functions and Dirichlet series in number theory, volume 41 of Graduate Texts in Mathematics. Springer-Verlag, New York, second edition, 1990.

[2] J.-B. Bost. Périodes et isogenies des variétés abéliennes sur les corps de nombres (d'après D. Masser et G. Wüstholz). Astérisque, (237):Exp. No. 795, 4, 115-161, 1996. Séminaire Bourbaki, Vol. 1994/95.

[3] A. Buium. Differential modular forms. J. Reine Angew. Math., 520:95-167, 2000.

[4] P. A. Griffiths. The residue calculus and some transcendental results in algebraic geometry, I, Proc. Nat. Acad. Sci. U.S.A., 55 (1966), 1303-1309.

[5] R. Hartshorne. Algebraic geometry. Springer-Verlag, New York, 1977. Graduate Texts in Mathematics, No. 52.

[6] J. Hilgert, D. Mayer, and H. Movasati. Transfer operator for $\Gamma_{0}(n)$ and the Hecke operators for the period functions of $\operatorname{PSL}(2, \mathbb{Z})$. Math. Proc. of the Cambridge Philosophical Society, 139(1), 2005,(math.NT/03032).

[7] K. Kato and S. Usui. Borel-Serre spaces and spaces of SL(2)-orbits. In Algebraic geometry 2000, Azumino (Hotaka), volume 36 of Adv. Stud. Pure Math., pages 321382. Math. Soc. Japan, Tokyo, 2002.

[8] F. Martin and E. Royer. Formes modulaires et périodes, Formes modulaires et transcendance, Sémin. Congr., Soc. Math. France, Vol. 12, 1-117, 2005.

[9] N. M. Katz. p-adic properties of modular schemes and modular forms. In Modular functions of one variable, III (Proc. Internat. Summer School, Univ. Antwerp, Antwerp, 1972), pages 69-190. Lecture Notes in Mathematics, Vol. 350. Springer, Berlin, 1973.

[10] S. Lang. Introduction to modular forms, volume 222 of Grundlehren der Mathematischen Wissenschaften [Fundamental Principles of Mathematical Sciences]. SpringerVerlag, Berlin, 1995. With appendixes by D. Zagier and Walter Feit, Corrected reprint of the 1976 original. 
[11] H. Movasati. Calculation of mixed Hodge structures, Gauss-Manin connections and Picard-Fuchs equations. To appear in the proceeding of the Sao Carlos conference at CIRM, 2004 (math.AG/0412235).

[12] H. Movasati. Moduli of polarized Hodge structures. Preprint, 2006.

[13] Y.V. Nesterenko and P. Philippon. Introduction to algebraic independence theory, volume 1752 of Lecture Notes in Mathematics. Springer-Verlag, Berlin, 2001. With contributions from F. Amoroso, D. Bertrand, W. D. Brownawell, G. Diaz, M. Laurent, Yuri V. Nesterenko, K. Nishioka, Patrice Philippon, G. Rémond, D. Roy and M. Waldschmidt, Edited by Nesterenko and Philippon.

[14] T. Sasai. Monodromy representation of homology of certain elliptic surfaces, J. Math. Soc. Japan, 26, No2 (1974) 296-305.

[15] V. V. Šokurov. Shimura integrals of cusp forms. Izv. Akad. Nauk SSSR Ser. Mat., 44(3):670-718, 720, 1980.

[16] M. Waldschmidt, Transcendance de périodes: État des connaissances, Advances in Mathematics, Vol 1, No. 2, 2006 (Proceedings of 11th symposium of the Tunisian mathematical society). 